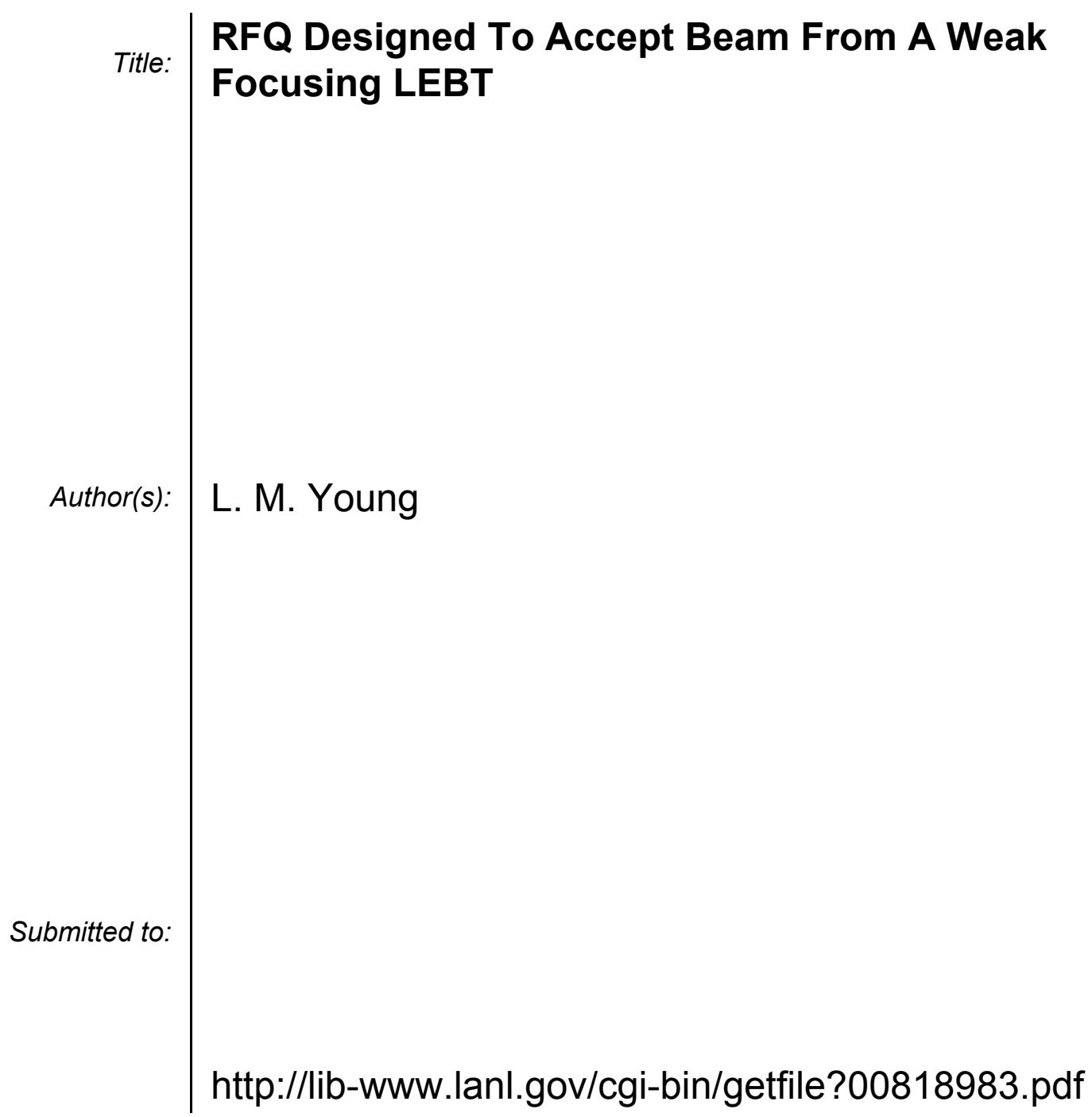

Los Alamos National Laboratory, an affirmative action/equal opportunity employer, is operated by the University of California for the U.S. Department of Energy under contract W-7405-ENG-36. By acceptance of this article, the publisher recognizes that the U.S. Government retains a nonexclusive, royaltyfree license to publish or reproduce the published form of this contribution, or to allow others to do so, for U.S. Government purposes. Los Alamos National Laboratory requests that the publisher identify this article as work performed under the auspices of the U.S. Department of Energy. Los Alamos National Laboratory strongly supports academic freedom and a researcher's right to publish; as an institution, however, the Laboratory does not endorse the viewpoint of a publication or guarantee its technical correctness. 


\title{
RFQ Designed To Accept Beam From A Weak Focusing LEBT*
}

\author{
L. M. Young \\ Los Alamos National Laboratory, Los Alamos, New Mexico 87545
}

\begin{abstract}
.
The LEDA RFQ is a 350-MHz continuous-wave $(\mathrm{CW})$ radio-frequency quadrupole linac. LEDA was designed as the full power front-end prototype for the accelerator production of tritium (APT) linac. This machine has accelerated a 100-mA CW proton beam from $75 \mathrm{keV}$ to $6.7 \mathrm{MeV}$. The 8-m-long RFQ accepts a dc, 75-keV, 110-mA H $\mathrm{H}^{+}$beam from the LEDA injector, bunches the beam, and accelerates it to full energy with $\sim 94 \%$ transmission. Output beam power is $670 \mathrm{~kW}$. This RFQ consists of four 2-meter-long RFQs joined with resonant coupling to form an 8-meter-long RFQ.
\end{abstract}

\section{INTRODUCTION}

The RFQ [1-5] receives a continuous stream of 75$\mathrm{keV}$ protons from the $\mathrm{H}^{+}$injector, [6,7] forms it into bunches with a high capture efficiency $(\sim 94 \%)$, and then accelerates these bunches to an energy of $6.7 \mathrm{MeV}$. Figure 1 shows the coupled RFQ structure mounted in the tuning laboratory. Figure 2 shows the RFQ structure configuration including tapered RF power feeds, vacuumport placement, and section nomenclature. Figure 3 shows a photograph of the completed RFQ assembly in the LEDA tunnel with the injector pulled back. The array of vacuum manifolds, water-cooling manifolds, and RF waveguide almost completely hides the accelerating structure.

\section{Design features}

With output energy of $6.7 \mathrm{MeV}$ the LEDA RFQ $[1,8]$ is the highest energy and highest power RFQ in the world $[3,5,9-11]$. The beam power is $670 \mathrm{~kW}$ when operated with the design-value $100-\mathrm{mA} \mathrm{CW}$ proton beam, making it the second-most powerful linear accelerator (after the LANSCE $800-\mathrm{MeV}$ linac). Some of its unique design features are as follows:

- Transverse focusing strength at the RFQ entrance is reduced for easier beam injection. This allows placement of the final focusing solenoid in the low energy beam transport (LEBT) at the optimum distance from the RFQ for input matching.

- It employs resonant coupling [12,13] between the four 2-m-long segments, providing high RF field stability throughout the entire structure length.

- It has a significantly larger aperture and gap voltage in the accelerating section than previously designed RFQs at this frequency.

- Transverse focusing at the RFQ exit is reduced to match the focusing strength in the next accelerating structure.

- $\quad$ RF power from three 1-MW klystrons is coupled to the RFQ through six waveguide irises. The structure itself combines the RF power.

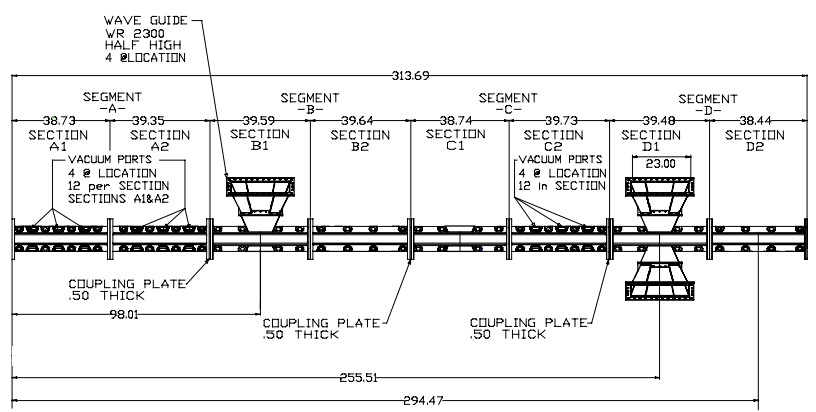

FIGURE 2: RFQ layout, showing RF feeds, vacuum ports, and segment nomenclature.

\section{Resonant Coupling}

In a typical RFQ that has constant focusing strength and constant gap voltage, as vane modulation increases to accelerate the beam, the aperture shrinks and beam can be lost on the vane tips. As the energy rises the cell length

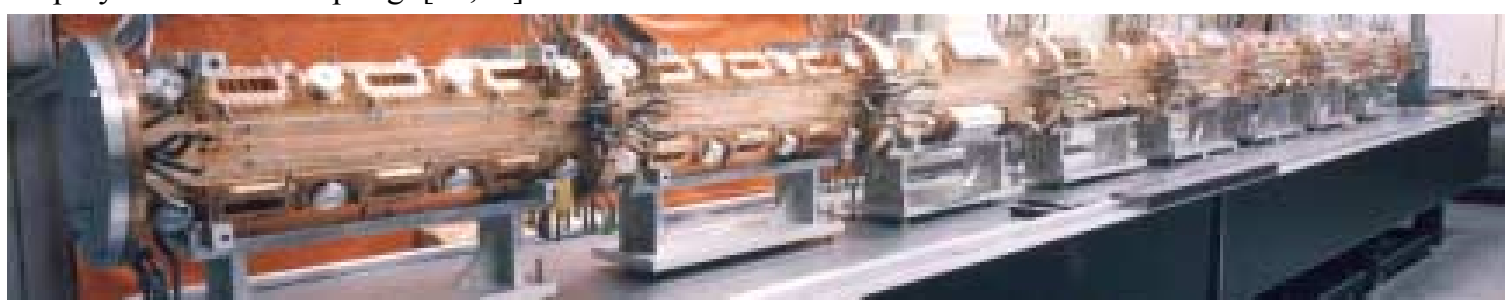

Figure 1. Eight-meter-long RFQ in the tuning laboratory. The adjustable slug tuners can be seen in this picture.

\footnotetext{
* Work supported by the US DOE, Defense Programs.

† lyoung@lanl.gov
} 
increases, and for a given modulation, the accelerating gradient decreases inversely with cell length. Since the maximum practical modulation is about 2, the RFQ would become very long if the gap voltage remained constant. To reduce beam loss and shorten the RFQ, we maintain a large aperture, and increase the vane voltage to partially counter the decrease in transverse focusing as the vane modulation increases.

The increased gap voltage substantially increases the accelerating field, thus shortening the RFQ. However, even with this increased gap voltage, eight meters of length is required to accelerate the beam to $6.7 \mathrm{MeV}$. A conventional 8-m-long, 350-MHz RFQ would not be stable. Small perturbations would distort the field distribution intolerably $[12,13]$. Therefore, four 2-m-long RFQs (labeled A, B, C and D in Figure 2) are resonantly coupled to form the 8-m-long LEDA RFQ. This is implemented by separating the four 2-m RFQs by coupling plates. An axial hole in the coupling plates allows the vane tips to nearly touch. The capacitance between the vane tips of one RFQ and the next provides the RF coupling between the 2-m-long segments. The gap between the vane tips at the coupling joint is $0.32 \mathrm{~cm}$. To minimize the effect of this gap on the beam, the gap position corresponds to a zero crossing of the RF electric field when the bunch passes the gap. The RF field is in phase in all four segments. The "coupling mode" has a strong electric field across the $0.32-\mathrm{cm}$ gap and has one longitudinal node in each 2-m RFQ. The coupling mode's longitudinal component of electric field transmits RF power, providing the field stability.

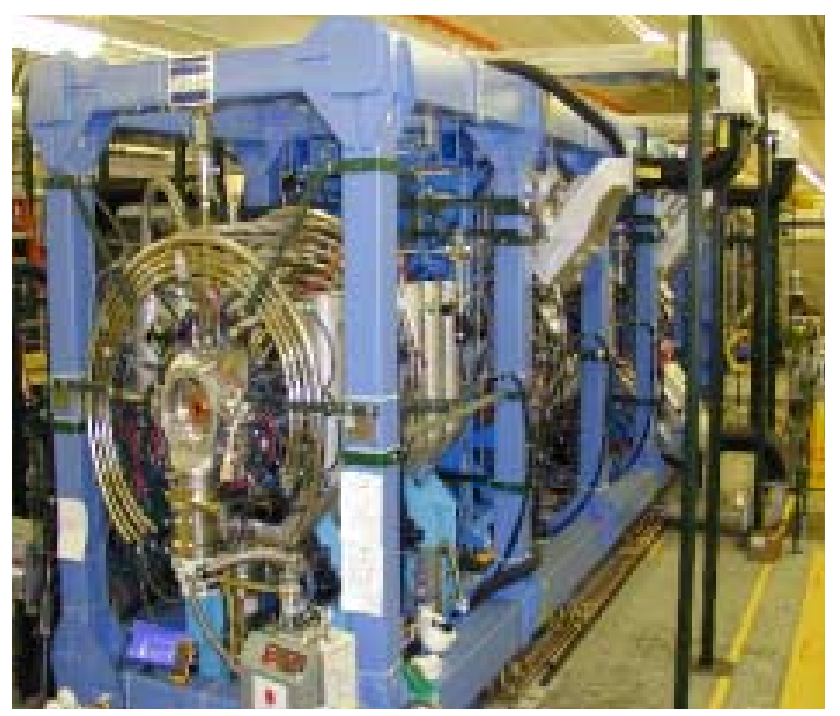

FIGURE 3. A photograph of the entrance end of the RFQ mounted in the support/alignment frame. The accelerating structure is buried within the array of water-cooling manifolds, vacuum manifolds, and RF waveguide.

\section{RFQ ELECTROMAGNETIC DESIGN}

The RFQ was designed with the code PARMTEQM (Phase and Radial Motion in Transverse Electric Quadrupole; Multipoles) [14]. The code includes the effect of higher-order multipoles in the RFQ fields, which are important in accurately predicting beam loss. The earlier code version, PARMTEQ, used only the first two terms in the expansion of the RFQ fields. PARMTEQM uses the first eight terms. In addition, a realistic description of the input beam is required to accurately simulate beam losses in the RFQ. Simulations of the beam transport through the LEBT [15] with PARMELA [16,17] produce a more realistic distribution of particles for input into the RFQ codes than the ideal input distributions.

\section{RFQ Entrance}

To implement the reduced focusing strength at the entrance of the RFQ and have adequate focusing in the interior of the RFQ, the transverse focusing parameter increases smoothly from 3.088 to 6.981 over the first 32 $\mathrm{cm}$ of the RFQ. The focusing parameter is proportional to $V / r_{0}{ }^{2}$ where $V$ is the voltage between adjacent vane tips and $r_{0}$ is the average aperture. The voltage is held constant in this region and the aperture is reduced to increase the focusing parameter. On entry, the beam is not yet bunched, allowing the use of weak transverse focusing. By the time the beam starts to bunch, the focusing is strong enough to confine the bunched beam.

The low focusing strength at the RFQ entrance means that the matched beam size is relatively large, allowing some space, as shown in figure 4, between the second LEBT solenoid and the RFQ entrance. Without this feature, the solenoid would be right at the RFQ entrance.

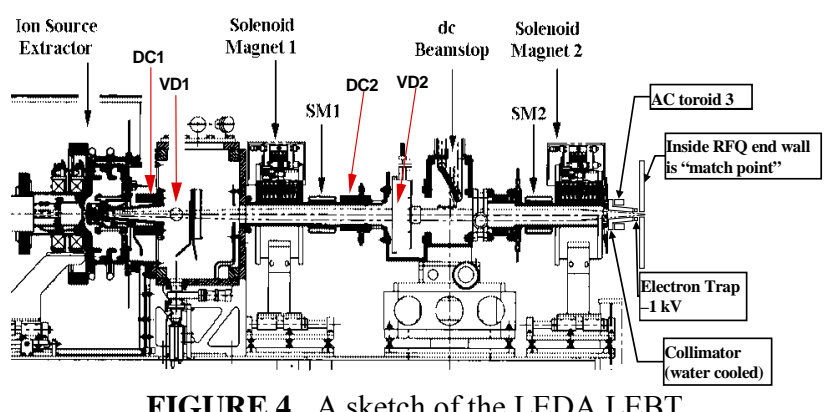

\section{TRANSMISSION THROUGH THE RFQ}

The code PARMELA simulates the LEBT beam with 95\% space-charge neutralization [18]. These simulations showed that proper matching would be possible with an electron trap at the RFQ entrance, and solenoid-to-RFQ distance of $15 \mathrm{~cm}$. The electron trap is a metal ring at the entrance of the RFQ. A ring voltage of $-1 \mathrm{kV}$ blocks lowenergy plasma electrons, but does not affect the $75-\mathrm{keV}$ protons. The electron trap performs two essential functions. One, it improves the space charge neutralization in the LEBT. Two, it prevents electrons from streaming into the RFQ through the torrid and corrupting the measurement of the beam current.

Using the simulated beam, two RFQ codes predict 93\% transmission with the RFQ operating at design field 
levels. The codes are PARMTEQM and TOUTITIS [19] that use respectively $2 \mathrm{D}$ and $3 \mathrm{D}$ space charge effects. The measured transmission has been as high as $94 \%$ at 100 $\mathrm{mA}$ when the RFQ fields are $10 \%$ above the design field strength.

\section{POSSIBLE ION TRAPPING IN RFQ}

Figure 5 shows the time dependence of RFQ transmission in a $300-\mu$ s-long beam pulse with the RFQ fields at the nominal design value. At about $150 \mu$ s into the pulse, the transmission suddenly drops by about $10 \%$. As the RFQ field is increased above the design value, transmission remains high for increasingly longer periods.

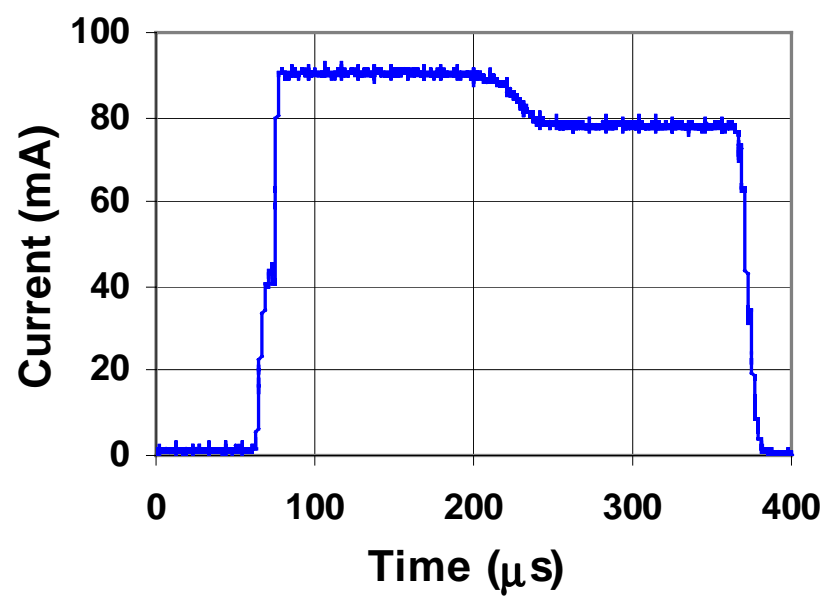

FIGURE 5. RFQ output beam current vs. time for a 300-mslong pulse at $\sim 97 \%$ of the design RF field level.

With fields $\geq 105 \%$ of design, the transmission drop is no longer observed, even for long pulses and $\mathrm{CW}$ operation. Along with the transmission drop, higher-than-expected activation is measured at the high-energy end of the RFQ, indicating significant beam loss at that location. Operating the RFQ with fields about $10 \%$ above the design value greatly reduces the magnitude of this beam loss. More work is needed to determine unambiguously the cause of the time-dependent transmission anomaly. At present, considerable evidence points to the possibility that it is caused by low-energy $\mathrm{H}^{+}$ions trapped near the axis by the RFQ fields [20]. The extra positive charge from the trapped ions causes the beam size to increase, reducing the RFQ transmission, and also increasing the beam size at the end of the HEBT. This hypothesis is consistent with the observation that the collimator ring in front of the beam stop glows, presumably from being struck by incident beam, when the RFQ pressure exceeds $1-2 \times 10^{-7}$ Torr. The low-energy $\mathrm{H}^{+}$ions can be produced by beam collisions with background gas $\left(\mathrm{H}_{2}\right)$ near the RFQ axis, or by beam collision with the vane tip surfaces. At fields $\leq$ the design value, the beam may be sufficiently large that its fringes strike the RFQ vane tips, creating $\mathrm{H}^{+}$ions that get trapped temporarily in the beam channel. As the trapped charge accumulates, the beam becomes larger still, until the transmission drops suddenly.

\section{REFERENCES}

1 D. Schrage et al., "CW RFQ Fabrication and Engineering," Proc. LINAC98 (Chicago, 24-28 August 1998) pp. 679-683.

2 L. M. Young et al., "High Power Operations of LEDA," Proc. LINAC2000 (Monterey, 21-25 August 2000) pp. 336-340.

3 L. M. Young et al., "Low-Energy Demonstration Accelerator (LEDA) Radio-Frequency Quadrupole (RFQ) Results," Proc. 2nd ICFA Advanced Accelerator Workshop on the Physics of HighBrightness Beams (Los Angeles, CA) pp. 488-503.

4 H. V. Smith, Jr. and J. D. Schneider, "Status Report on the LowEnergy Demonstration Accelerator (LEDA)," Proc. LINAC2000 (Monterey CA, 21-25 August 2000) pp. 581-3.

5 L. J. Rybarcyk et al., "LEDA Beam Operations Milestone and Observed Beam Transmission Characteristics," Proc. LINAC2000 (Monterey CA, 21-25 August 2000) pp. 584-6.

6 T. Zaugg, C. Rose, J. D. Schneider, J. Sherman, R. Stevens, Jr., "Operation of a Proton Microwave Source in Pulsed Mode," Proc. LINAC98 (Chicago, 24-28 August 1998) pp. 893-95.

7 J. D. Sherman et al., "Status Report on a dc 130-mA, 75-keV Proton Injector," Rev. Sci. Instrum. 69 (1998) 1003-8.

8 J. D. Schneider, "Operation of the Low-Energy Demonstration Accelerator: the Proton Injector for APT," Proc. PAC99 (New York, 29 March - 2 April 1999) pp. 503-507.

9 H. V. Smith, Jr. et al., "Update on the Commissioning of the LowEnergy Demonstration Accelerator (LEDA) Radio-Frequency Quadrupole (RFQ)," Proc. 2nd ICFA Advanced Accelerator Workshop on the Physics of High-Brightness Beams (Los Angeles, CA) pp. 466-479.

10 D. E. Rees et al., "Design, Operation, and Test Results of 350 MHz LEDA RF System," Proc. LINAC98 (Chicago, 24-28 August 1998) pp. 564- 566.

11 K. F. Johnson et al., "Commissioning of the Low- Energy Demonstration Accelerator (LEDA) Radio- Frequency Quadrupole (RFQ)," Proc. PAC99 (New York, 29 March - 2 April 1999) pp. 3528-3530.

12 M. J. Browman and L. M. Young, "Coupled Radio- Frequency Quadrupoles as Compensated Structures," Proc. of the 1990 Linear Accelerator Conference, (Albuquerque, 10-14 Sept. 1990) LA12004-C, pp. 70-2.

13 L. M. Young, "An 8-meter-long Coupled Cavity RFQ Linac," Proc. 1994 Int. Linac Conf., (Tsukuba, 21-26 Aug. 1994) pp. 178180.

14 K. R. Crandall et al., "RFQ Design Codes," Los Alamos National Laboratory report LA-UR-96-1836 (Revised August 21, 1998).

15 H. V. Smith, Jr. et al., "Comparison of Simulations with Measurements for the LEDA LEBT H+ Beam," Proc. PAC99 (New York, 29 March - 2 April 1999) pp. 1929-31.

16 L. M. Young, "PARMELA," Los Alamos National Laboratory report LA-UR-96-1835 (Revised February 27, 2001).

17 L. M. Young, "Simulations of the LEDA LEBT With H+, H2+, and e- Particles," Proc. of the 1997 Particle Accelerator Conference (Vancouver, 12-16 May 1997) pp. 2749-2751.

18 Robin Ferdinand Joseph Sherman, Ralph R. Stevens, Jr., Thomas Zaugg, "Space-Charge Neutralization Measurement of a 75-Kev, 130-mA Hydrogen-Ion Beam,” Proc. PAC97 (Vancouver, B.C., Canada 12-16 May 1997) pp. 2723-2725.

19 R. Duperrier, R. Ferdinand, J-M, Lagniel, N Pichoff, "Toutatis, The CEA-SACLAY RFQ Code," Proc. LINAC2000 (Monterey, 21-25 August 2000).

20 M. S. deJong, "Background Ion Trapping in RFQs," Proc. 1984 Linac Conf. (Seeheim, Germany, 7-11 May 1984), pp. 88-90. 